\title{
COVID-19: la bomba sulla salute e sulla Sanità. Ricostruiamo da lì
}

\author{
Marilena Cara ${ }^{1}$, Alessandro Toccafondi ${ }^{2}$, Roberto Russo ${ }^{3}$, Marco Lombardi ${ }^{4}$ \\ ${ }^{1}$ Medico Nefrologo Psicologo Analista, socioanalista C.I.P.A. e I.A.A.P., Libero professionista, Padova - Italia \\ ${ }^{2}$ SOSD Psicologia Clinica in servizio presso SOC Nefrologia e Dialisi Firenze 2 ASLTC, Firenze - Italia \\ ${ }^{3}$ UO Nefrologia Dialisi Trapianto, Azienda Ospedaliera Policlinico di Bari, Bari - Italia \\ ${ }^{4}$ SOS Nefrologia e Dialisi, ASLTC Ospedale del Mugello, Borgo San Lorenzo, Firenze - Italia
}

Non siamo in grado di dire ciò che accadrà nel nostro prossimo futuro. Quello che sappiamo è che l'incertezza e la paura occupano prepotentemente il nostro presente.

L'unica certezza è che questa guerra ha colpito la salute. La bomba sganciata sul nostro punto vitale ha avuto un effetto domino, colpendo la Sanità, l'economia e, di conseguenza, la nostra sussistenza.

L'inizio dell'epidemia ha fatto risuonare nelle strade e nelle case la voce dei camici bianchi, fino ad allora sommessa e nascosta.

Il tempo della Sanità prima del COVID ha troppo spesso raccontato delle ombre, un tempo quasi come ritmato dal suono dei tamburi della malasanità: ad ogni rintocco seguivano un'accusa, un "colpevole" e un "approfittatore" del posto statale che veniva stanato.

Stiamo vivendo una frattura epocale, una crisi: la crisi della Sanità.

Questo è il momento per ricominciare con nuove prospettive e, se così non fosse, tutte le cose che oggi si sostengono sui nuovi "eroi" finirebbero per rivelarsi solo parole del momento e una dolorosa ipocrisia.

Prendersi cura della salute, che è il nostro bene più prezioso, racchiude un senso di coraggio e di responsabilità che non si esaurisce nelle parole, ma si concretizza in un processo diagnostico e terapeutico che deve portare a dei risultati tangibili: guarire e curare la malattia e il dolore.

Coloro che gestiscono la Sanità rappresentano il pilastro fondante della nostra collettività e la pandemia ce lo insegna. II COVID ha messo con le spalle al muro sanitari e custodi della Sanità, obbligandoli a una scelta, "La Scelta": ripristinare

Received: April 16, 2020

Accepted: April 16, 2020

Published online: May 6, 2020

Indirizzo per la corrispondenza:

Marilena Cara

via Cellini 4

Noventa Padovan, Padova - Italia

marilena.cara@libero.it la vecchia Sanità o farla evolvere, facendo crescere un nuovo modo per prendersi cura di chi cura e, quindi, della salute collettiva.

I medici, gli infermieri e gli operatori sanitari hanno dato la vita per la collettività, dimostrando a tutti l'importanza dei valori umani, al di là di uno stipendio e della svalutazione derivante dall'essere trattati come numeri e non come persone.

La psicanalisi, che studia il funzionamento psichico, permette di prevedere cosa potrebbe accadere domani alle persone che, oggi, lavorano negli ospedali e curano i pazienti COVID e vivono nei reparti COVID.

Le persone che oggi vivono il carico emotivo della cura estrema si troveranno domani ad affrontare un'area traumatica di cui oggi non sono ancora consapevoli.

Il trauma è ciò che spezza lo scorrere ordinario delle nostre vite, è guardare l'inguardabile.

Da medico (CM) che ha vissuto i reparti ospedalieri, posso permettermi di affermare che queste persone attualmente passano le loro 36 ore settimanali e più a contatto stretto con la morte tangibile, con la sofferenza, con il sentire sulla pelle la mancanza di respiro del paziente che sta morendo di fronte ai loro occhi, con il dolore di dover comunicare un decesso. Conosco bene quella sofferenza dell'Anima, quando, nella notte, ti torna alla mente l'ultimo sguardo del tuo paziente che ti chiede di non abbandonarlo, quando ti risuona nella mente il silenzio angosciante che irrompe nella telefonata in cui avvisi della morte di un padre, di una moglie o di un figlio.

So cosa significa accompagnare una persona alla morte. Constatare un decesso è l'ultimo atto medico, un atto fatto di dolore, di mistero, di incredulità. Quante volte ho sentito i colleghi dire: "quando constato un decesso controllo sempre più volte tutti i parametri vitali perché ho la sensazione che il paziente si possa risvegliare". Rimani accanto all'ultima espressione del suo volto, la sua vita e la sua Anima ti scorrono nella mente come un film. Fare il medico, ancor più essere medico, è un lavoro emotivamente faticoso e profondo, di cui non ci se rende neppure conto, perché non si ha o non si vuole avere il tempo per pensare questa cosa. Ricordo che, dopo la constatazione di morte, chiedevo di essere lasciata sola nella stanza con il mio paziente per pensarlo ancora 
una volta, cosi mi ha insegnato un grande padre spirituale. Accompagnare alla morte è un'esperienza che intacca l'Anima. Dall'esterno tutto appare normale routine, perché sai che devi andare avanti a curare altre persone, devi procedere a fare il tuo dovere e non puoi permetterti di cedere ai pensieri e alle emozioni.

Possiamo immaginare cosa accade nell'Anima e nella psiche di tutte le persone che hanno visto morire un paziente dopo l'altro con la sensazione di grande impotenza? Possiamo immaginare quanto difficile sia per un medico fare i conti con l'impotenza che dilaga di fronte a una strage? Possiamo immaginare il dolore di un'infermiera infettata da COVID nel vedere i propri familiari infettati a causa sua o, peggio, nel vedere il proprio padre morire di COVID sapendo che sei tu la causa di contagio?

La psicologia conosce ciò che accade all'Anima, ma il medico lo vive. Le due prospettive non possono più rimanere separate. La teorizzazione e il vissuto non possono più rimanere disgiunti.

La psicologia conosce bene la teoria della dissociazione psichica di cui i sanitari soffrono, ma forse non tutti l'hanno vissuta.

Per quanto l'empatia possa venire in aiuto alla comprensione emotiva dell'altro, sappiamo bene, noi analisti, che la vita clinica del terapeuta è altrettanto fondamentale. Dissociare un'emozione senza rendersene conto perché nessuno ti hai mai illustrato cosa sia è un processo praticamente automatico di fronte alla morte di un tuo paziente e alla sofferenza scolpita sul suo volto e su quello dei familiari, alla paura di essere contagiati e alla paura di morire come loro, i tuoi pazienti.

Piccoli momenti di dissociazione mentale sono necessari per poter affrontare il dolore e la paura. I sanitari, ma, in particolar modo, i medici che hanno il peso della responsabilità ultima del paziente, non hanno, generalmente, consapevolezza di questo, non possono averla, non è mai stata loro illustrata. Chi ha vissuto il COVID da vicino si troverà ad affrontare vissuti emotivi e ricordi intrusivi di questa esperienza.

La psicologia medica dovrebbe essere la nuova formazione per la nuova Sanità fatta da psicoterapeuti esperti di Sanità, che sappiano unire la psicologia alla medicina.

La psicologia medica dovrebbe essere parte integrante della nuova Sanità che tutti noi siamo chiamati a costruire. In questo processo, sarà fondamentale il contributo di psicologi e psicoterapeuti esperti di Sanità e delle implicazioni psicologiche e psicopatologiche nei contesti sanitari, figure che sappiano unire la psicologia alla medicina.

Possiamo pensare alla creazione di gruppi di lavoro di professionisti con formazione ed esperienza specifica in ambito sanitario e nell'area traumatico-dissociativa, coordinati e supervisionati da linee comuni di intervento, che realizzino in tempi brevi una formazione sul campo, attraverso gruppi di lavoro e lezioni teoriche, al fine di rimarginare diverse fratture: per esempio, il senso di colpa per avere perso un familiare dopo averlo contagiato con il COVID, i ricordi di morte e sofferenza che risalgono a un periodo concentrato nel tempo, il lutto dei colleghi morti sul campo, la suggestione corporea (senso di dispnea, ansia, disturbi intestinali, dolori muscolari) che potrebbe continuare a esistere, il persistere della paura di riprendere un contatto fisico con i familiari. Parte del progetto dovrebbe comprendere anche colloqui individuali laddove fosse necessario.

Purtroppo, la mentalità medica italiana è ancora troppo poco abituata a considerare l'importanza degli aspetti emotivi nella formazione delle persone che vivono sotto i camici bianchi, ma sarà indispensabile ripartire da nuove prospettive.

La relazione con i malati, con la sofferenza, con la morte, il confronto con l'impotenza, la rabbia, l'angoscia. Per coloro che si occupano della cura delle sofferenze umane il rapporto con questi temi è ordinario e costante.

Tuttavia, raramente noi medici siamo stati formati ad affrontare, interiormente, pensieri ed emozioni di questa portata, ritrovandoci da soli con una sofferenza a cui nessuno ci ha insegnato a dare un nome. In questo senso, è necessario che queste tematiche diventino sempre più parte integrante del percorso formativo di base e specialistico del medico, così come di tutte le altre figure sanitarie.

Ci rivolgiamo a coloro che sono in prima in linea e, soprattutto, a coloro che hanno la responsabilità di amministrare la nuova Sanità. Potrebbe essere l'inizio di una nuova medicina.

Operatori, infermieri e medici prima di tutto sono uomini, non eroi estemporanei.

Non lasciamoli soli nella nebbia.

\section{Disclosures}

Conflict of interest: The authors declare that there is no conflict of interest.

Financial support: The authors declare no financial support. 